\title{
Prevalencia de bullying en estudiantes de una secundaria pública del municipio de Aguascalientes
}

González Ávila Jazmín Lucia, ${ }^{*}$ Guerrero Mojica Nery**

\section{- Resumen}

- Introducción. El bullying es un problema de salud que se pre-

- senta entre los adolescentes, se caracteriza por un compor-

- tamiento agresivo donde existe una relación interpersonal

- basada en una asimetría de poder y se manifiesta como

- una agresión física y verbal, por medio de insultos, amenazas,

- intimidación, el chantaje, el aislamiento, la exclusión social,

- golpes y el acoso racial de alguien que se considera fuerte

- hacia otro que es débil; llegando a originar un desequilibrio

- en el estado físico y mental de la persona que es víctima de

- acoso escolar. Objetivo. Establecer la prevalencia del bullying

- en estudiantes de una secundaria pública del municipio de

- Aguascalientes. Metodología. Estudio descriptivo y transver-

- sal con una muestra de 153 estudiantes del turno vesperti-

- no de una secundaria pública, en quienes se aplicó el auto

- test de Cisneros con un Alpha de Crombach de 0.914 para

- la escala total de 50 reactivos. Resultados. El 54.2\% de los

- estudiantes fueron hombres y el $45.8 \%$ mujeres, con una

- edad promedio de 13.62 años, con respecto al grado escolar

- el $34.6 \%$ lo ocupan el segundo y tercero de secundaria. El

- $42.5 \%$ de los estudiantes manifestaron bullying, el tipo de

- acoso más frecuente fue la coacción con un 90.9\%. Conclu-

- sión. La presencia de bullying en los estudiantes de secunda-

- ria tiene una alta prevalencia, lo cual puede desencadenar

- serios problemas en la salud e incluso la muerte. LUXMÉDICA,

- AÑO 11, NÚMERO 34, SEPT-DIC 2016, PP 19-24

Palabras clave: Prevalencia, bullying, estudiantes de

secundaria

$\bullet$

$\bullet$

\section{Abstract}

Introduction: Bullying is a health problem that occurs among teenagers. It is characterized by aggressive behavior where there is an interpersonal relationship based on asymmetry of power and it manifests itself both physically and verbally: through insults, threats of aggression, intimidation, blackmail, isolation, social exclusion, violence, and the racial harassment of someone who is considered strong towards one that is weak. This results in an imbalance in the physical and mental state of the person who is the victim of bullying at school. Objective. Establish the prevalence of bullying in students of a public secondary school of the municipality of Aguascalientes. Methodology. Descriptive and cross-sectional study with a sample of 153 students from the evening shift from a public secondary school, who applied the self- test of Cisneros with a Cronbach's Alpha of 0.914 for the total scale of 50 items. Results. $54.2 \%$ of students were men and the $45.8 \%$ women, with an average age of 13.62 years, with respect to the grade level $34.6 \%$ were from the second and third secondary school grade. $42.5 \%$ of students reported bullying, more frequent harassment was coercion with a $90.9 \%$. Conclusion. The presence of bullying in secondary school students has a high prevalence, which can trigger serious problems in the health and even death. LUXMÉDICA, AÑO 11, NÚMERO 34, SEPT-DIC 2016, PP 19-24

Keywords: prevalence, bullying, secondary school students

*Pasante del servicio social de la carrera de Enfermería del Centro de Ciencias de la Salud de la Universidad Autónoma de Aguascalientes. Correo electrónico: hugo2_03@hotmail.com

**Profesora investigadora de la Licenciatura en Enfermería del Centro de Ciencias de la Salud de la Universidad Autónoma de Aguascalientes. Correo electrónico: g.n.ery@hotmail.com

Fecha de recibido: 4 de junio 2016

Fecha de aceptación: 13 de agosto 2016

Correspondencia: Nery Guerrero Mojica, Departamento de Enfermería, Centro de Ciencias de la Salud. Universidad Autónoma de Aguascalientes. Avenida Universidad N 940, Edificio 105 Ciudad Universitaria. Código postal. 20131. Teléfono (449) 9108437. Aguascalientes, Ags. México. Correo electrónico: g.n.ery@hotmail.com 


\section{Introducción}

La Organización para la Cooperación y el Desarrollo Económicos (OCDE) sitúa a México en el primer lugar de bullying o acoso escolar de los países que integran este organismo (34 países). ${ }^{1}$ La violencia que ocurre en los contextos educativos se denomina violencia escolar y se define como "cualquier acción u omisión intencional que en la escuela, alrededores de la escuela o actividades extraescolares, daña o puede dañar a terceros". ${ }^{2}$ Se considera un problema de salud pública que se presenta con mayor frecuencia entre los adolescentes que se encuentran en educación básica. ${ }^{3}$ Ha sido considerado para muchos como una falsa creencia de que es algo natural y propio de la edad, que se presenta como una forma de relacionarse entre los que lo padecen y sin darse cuenta en realidad de la problemática y de las consecuencias que pueden originarse cuando alguien vive esta situación de violencia. ${ }^{4}$ Según la Organización Mundial de la Salud, define la adolescencia como la etapa que transcurre entre los 11 y 19 años, donde se presentan cambios tanto en el aspecto fisiológico, cambios estructurales anatómicos y modificación en el perfil psicológico y de la personalidad. ${ }^{5}$

El bullying es una agresión ya sea física o verbal, que se manifiesta a través de insultos, amenazas, intimidación, el chantaje, el aislamiento, la exclusión social, golpes y el acoso racial de alguien que se considera fuerte hacia otro que es débil ${ }^{3}$ presentándose de forma permanente durante un periodo de tiempo llegando a provocar en la víctima temor por los actos que se le ocasionan sin que las autoridades encargadas de las instituciones públicas, maestros y padres de familia se den cuenta de lo que pasa, manteniéndose en secreto y que, eventualmente, esto puede ocasionar problemas de salud entre los que lo sufren, como bajo rendimiento escolar, baja autoestima, estrés, lesiones físicas, depresión, trastornos alimenticios, consumo de drogas y suicidios por el temor de no poder enfrentar y soportar la situación en la que están viviendo. ${ }^{5}$

Smith y Sharp son de los primeros autores en proponer un concepto general de lo que es el "bullying" y lo definen como una relación cotidiana entre varias personas en la que se ejerce un abuso sistemático del poder, sin especificar el sitio en el que se desarrolla el fenómeno. ${ }^{6}$ Narváez lo definió como la forma en que un alumno es agredido de forma física y psicológica o se convierte en víctima cuando está expuesto, de forma repetida y durante un tiempo a acciones negativas que lleva acabo a otro alumno o varios de ellos. ${ }^{7}$

Se estima que cada año se suicidan en el mundo alrededor de 600 mil personas entre los 14 y 28 años, cifra que por lo menos, la mitad, tiene alguna relación con el bullying y afectando más a los países europeos en el siguiente orden como Reino Unido, Rusia, Irlanda, España e Italia al obtener 200 mil suicidios al año según datos obtenidos de la Organización Mundial de la salud (OMS) y la Organización de las Naciones Unidas (ONU). ${ }^{3}$ 
El bullying es un fenómeno complejo que por su naturaleza conlleva consecuencias negativas para todos los actores que están involucrados. ${ }^{8}$ Cuando se vive acoso escolar se dificulta el aprendizaje de los estudiantes, les causa daños físicos y psicológicos a las víctimas y el involucramiento en el bullying es un factor de riesgo que incrementa las probabilidades de emprender trayectorias problemáticas; ${ }^{9}$ además se relaciona con autoestima baja y con sentimientos de soledad a largo plazo, teniendo repercusiones en la vida futura de los individuos.

Cuando existe una exposición crónica a los patrones de convivencia violentos, todos los involucrados, víctimas y agresores, tienen mayor riesgo de sufrir síntomas depresivos e ideación suicida, pasando por trastornos del sueño, dolor abdominal, cefalea, malestar general, ansiedad, baja autoestima, sensación de rechazo social, aislamiento, marginación y una autopercepción de minusvalía, física y social. ${ }^{10}$

El objetivo de la investigación fue conocer la prevalencia de acoso escolar en estudiantes de una secundaria pública del municipio de Aguascalientes.

\section{| | | | | | | | | | | | | | | | | | | | | | | | | | | | | | | | | | | | | | | | | | | | | | | | | | | | | | | | | | | | | | | | | | | | | | | | | | | | | | | | | | | | | | | | | | | | | | | | | |||}

\section{Material y métodos}

Se realizó un estudio descriptivo, prospectivo y transversal. El universo estuvo conformado por 253 estudiantes del turno vespertino de una secundaria pública del municipio de Aguascalientes. Se obtuvo una muestra de 153 estudiantes por muestreo aleatorio simple, con un margen de error de $5 \%$ y un nivel de confianza del $95 \%$. Se incluyeron a los estudiantes de primero, segundo y tercer grado escolar. Se aplicó el auto test de Cisneros de acoso escolar que evalúa el índice global de acoso escolar elaborado por Iñaki Piñuel y Araceli Oñate a través del Instituto de Innovación Educativa y Desarrollo Directivo en el año 2005 en España, con un índice de confiabilidad alpha de Crombach de 0.914 . Consta de 50 ítems en los que los escolares marcan la frecuencia con la que son objeto de distintas modalidades de maltrato. Tiene tres posibilidades de respuesta nunca, pocas veces y muchas veces asignándose puntajes de 1, 2 y 3 puntos respectivamente. El cuestionario presenta 10 escalas de las que ocho corresponden a diferentes tipos de acoso y dos que se refieren respectivamente al "Índice global de riesgo y a la intensidad del maltrato". Los tipos de maltrato que evalúa son: intimidaciónamenazas, coacción, desprecio-ridiculización, exclusión -bloqueo social, restricción- comunicación, robos, hostigamiento verbal y agresiones. ${ }^{11}$ Se elaboró una base de datos en el SPSS versión 2.1 para calcular a estadística descriptiva y obtener la calificación de cada rubro que mide el test por medio de la técnica de baremos globales, de acuerdo a sus categorías bajo, medio, alto y muy alto.

\section{|| || || || || |||||||||||||||||||||||||||||||||||||||||||||||||||||||||||||||||||||||||||||||||||||||||||||||||||||||||||||||}

\section{Resultados}

En la investigación participaron 153 estudiantes de los cuales 83 fueron hombres
(54.2\%) y 74 mujeres (45.8\%). La edad promedio fue de $13.6 \pm 1.0$ años, con una edad mínima de 12 años y una máxima de 
17 años, predominando los de 14 años con un $33.3 \%$ de los adolescentes. Respecto al grado escolar el $34.6 \%$ lo ocupa el segundo y tercer grado respectivamente.

Para obtener el índice global de acoso escolar se realizó la suma total de los 50 reactivos que conforman el instrumento, de acuerdo al valor de cada ítem $(1,2$ y 3 puntos) y se dividió en cuatro categorías: bajo, con 50 puntos; medio, si puntuación entre 51-58; alto, con 59-68 puntos; y muy alto, con puntuación entre 89-150. En la figura 1 se muestra a los estudiantes que son víctimas de acoso escolar, podemos observar que el acoso escolar tipificado como alto, fue el de mayor frecuencia $(42.5 \%)$, medio en el $22.2 \%$, bajo en el $29.4 \%$ y el bullying considerado como muy alto, lo identificamos en el $5.9 \%$ de los estudiantes encuestados.

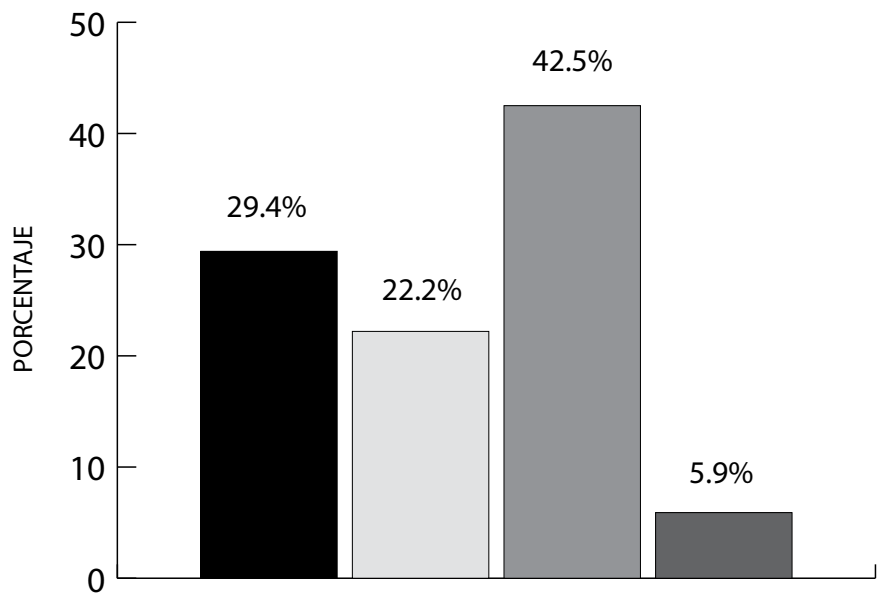

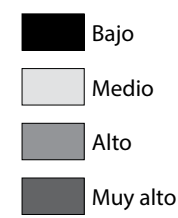

Figura 1. Distribución de la frecuencia del acoso escolar, por grado de severidad, en estudiantes de una secundaria pública del municipio de Aguascalientes. $n=153$ estudiantes
En la figura 2 se observa la intensidad en la que los estudiantes de la secundaria reciben la fuerza del maltrato, dividiéndose en las categorías correspondientes, bajo de 0-1, alto de 2-10 y muy alto de 11-50. La mayoría de los adolescentes (84.3\%) manifestó una intensidad baja del acoso escolar, seguida por intensidad alta $(11.8 \%)$ y muy alta $(3.9 \%)$.
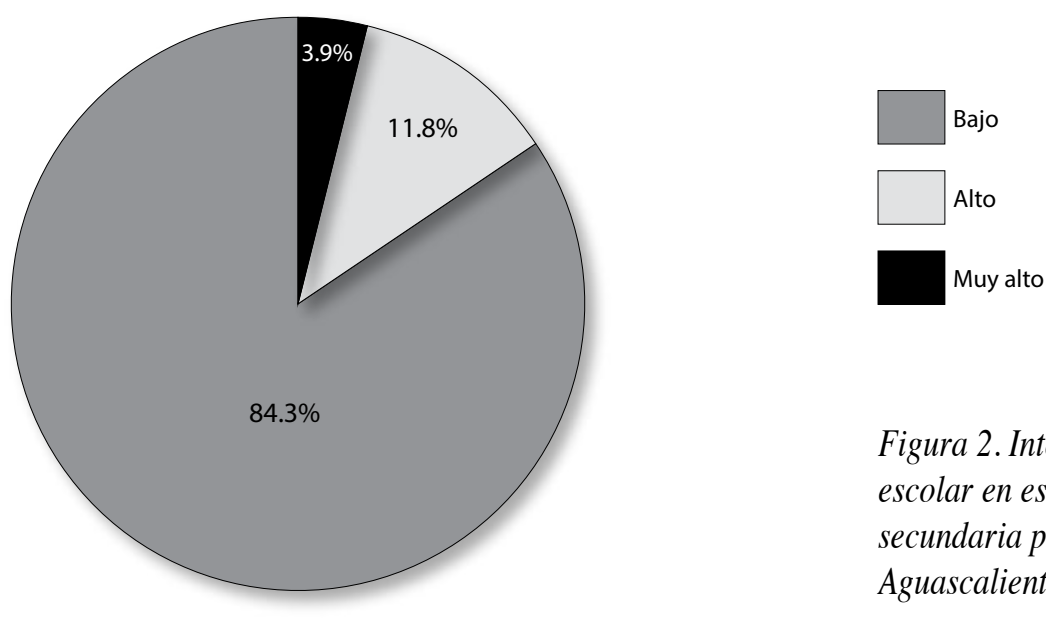

Figura 2. Intensidad de acoso escolar en estudiantes de una secundaria pública del municipio de Aguascalientes. 
El test de Cisneros identifica cuatro tipos de acoso: el físico que corresponde a agresiones y robos; el psicológico con intimidación, amenazas y coacción; el social con desprecio, restricción y exclusión social; y en el acoso verbal con hostigamiento verbal.

El tipo de maltrato más frecuente en los estudiantes fue el psicológico con la coacción en el $90.9 \%$ de los casos, es decir que representa aquellas conductas de acoso escolar que pretenden que el adolescente realice contra su voluntad. Mediante estas conductas, quienes acosan al adolescente pretenden ejercer un dominio y un sometimiento total de su voluntad.

La exclusión y la intimidación fueron los tipos de acoso escolar tipificadas con intensidad baja en el $85.1 \%$ y $60 \%$ respectivamente, como se puede observar en la figura 3.

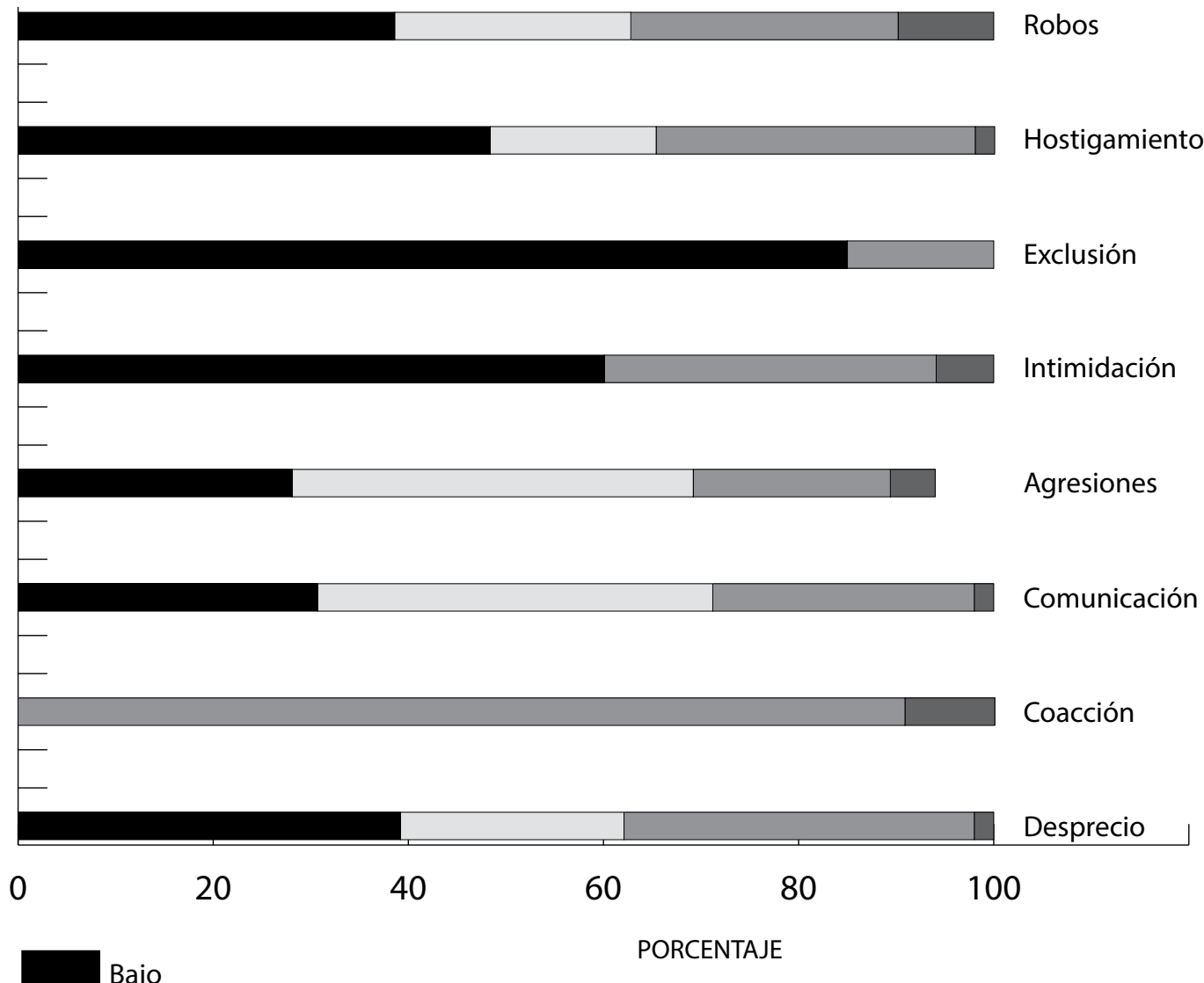

Figura 3. Tipos de acoso escolar, distribuidos por intensidad en estudiantes de una Medio secundaria.

Alto

Muy alto

Discusión

Las edades de los estudiantes participantes en el presente estudio oscilan entre los 12 y 17 años y se encuentran dentro de las edades que presentan mayor prevalencia de acoso escolar, según Hernández. ${ }^{12}$ De igual manera en estudiantes entre 12 y 16 años presentaron los porcentajes más elevados de bullying en algunos países como en Turquía con $30 \%$ en Corea con $28 \%$ 
y Estados Unidos con $24 \% . .^{13}$ Valadez en el año 2011 mencionó que independientemente del tipo de sistema educacional, el ambiente escolar tiene un gran impacto en la vida de los sujetos jóvenes, debido a la cantidad de tiempo que los estudiantes pasan en las escuelas así como por el tipo de interacciones sociales que presentan. ${ }^{14}$ En comparación con Morales y Ramírez en su estudio la prevalencia es muy alta con el $40.9 \%$, seguido de $31.4 \%$ con baja y $13.64 \%$ es alta, en cambio los datos ob- tenidos en esta investigación la prevalencia es alta con un el $42.5 \%$, el $29.4 \%$ es bajo y solamente el $5.9 \%$ es muy alta. ${ }^{15}$ Estos resultados están reflejando un serio problema de salud.

La violencia psicológica es el tipo de maltrato que más se vive entre los estudiantes investigados ya que el $90.9 \%$ lo presenta alto en coacción que es congruente con lo reportado por Hernández, que encontró a la violencia psicológica como la forma más común de agresión en los estudiantes con un $75.5 \%$. ${ }^{12}$

\section{Conclusiones}

La prevalencia de bullying en los estudiantes de una secundaria pública del municipio de Aguascalientes es alta, y la violencia psicológica fue la de mayor prevalencia. Una de las funciones fundamentales de la enfermería comunitaria sería colaborar con un equipo multidisciplinario en una estrategia destinada a disminuir, controlar y prevenir el acoso escolar.

\section{Bibliografía}

1. Organización para la Cooperación y el Desarrollo Económicos. Disponible en: https://www.oecd.org/centrodemexico/estadisticas/

2. Sanmartín, J. (2006). Concepto y tipos. En A. Serrano (Ed.): Acoso y violencia en la escuela. Cómo detectar, prevenir y resolver el bullying, (pp. 21-31). Barcelona:Ariel/Centro Reina Sofía para el estudio de la violencia.

3. Luciana Cataldi. Programa Mediación y Violencia "Bullying: Según las últimas estadísticas relación entre los casos de acoso escolar y el suicidio juvenil" 2013 Madrid España. Disponible en: http://mediacionyviolencia.com.ar/bullying-segun-las-ultimas-estadisticashay-realacion-entre-los-casos-de-acoso-escolar-y-elsuicidio-juvenil/

4. Carrión JJ, Gázquez JJ, Pérez MC, Clima escolar y resolución de conflictos según el alumnado: un estudio europeo. Revista de Psicodidáctica 2011;16:39-58. Disponible en: http://www.redalyc.org/articulo. oa?id=17517217003. Fecha de consulta: 11 de octubre de 2016.

5. Organización Mundial de la Salud. Salud de la madre, el recién nacido, del niño y del adolescente. Consultado en 16 de octubre de 2016. Disponible en: http:// www.who.int/maternal_child_adolescent/topics/ adolescence/dev/es/

6. Smith, P. Bullying in schools: Lessons from two decades of research. Aggresive Behav 2009; 26:1-9.

7. Narváez, V. Salazar, F. Carta de la salud. Bullying, ma- toneo, intimidación o acoso escolar, México 2012; 200: 1-4

8. Díaz, J. Martínez, M. y Vásquez, L. Una educación resiliente para prevenir e intervenir la violencia escolar. Itinerario Educativo. 2011; 57:121-155.

9. Collell, J. Carme, M. Armario de psicología clínica y de la salud. El acoso escolar: Un enfoque psicopatológico. España 2010; 2: 9-14.

10. Loredo, A. Perea, A. López, G. Bullying: acoso. La violencia entre iguales. Problema real en adolescentes. Acta pediátrica, México 2010; 29:4,210-4.

11. Informe Cisneros VII. Auto- test Cisneros. 2011. [Internet] (Consultado 2016 enero 10) Disponible en: http://www.conflictoescolar.es/2011/07/informecisneros-vii/

12. Hernández, E. Santos, G. Presencia de violencia entre estudiantes de una escuela secundaria publica de Coatzacoalcos Veracruz, [Tesis pregrado], Minatitlán, Veracruz. 2010 pág. 1- 36.

13. Pérez, Ma. Gázquez, J. Fernández, R. y del Mar, M Análisis de las publicaciones sobre convivencia escolar en una muestra de revistas de educación en la última década. Aula Abierta. 2011; 39:2, 81-90.

14. Valadez, R. Amezcua, N. González, R. Montes V. Maltrato entre iguales en sujetos escolarizados. Revista latinoamericana de ciencias sociales, Niñez y Juventud. 2011; 2:9,786-796.

15. Morales, Abigail. Bullying y su relación con la depresión en adolescentes. [Tesis pregrado], Universidad de Querétaro, 2014 pág. 1-85. 